\title{
Modeling information exchange between living and artificial cells
}

\author{
Keith C. Heyde ${ }^{1, \dagger}$, MaryJoe K. Rice ${ }^{2, \dagger}$, Sung-Ho Paek ${ }^{3}$, Felicia Y. Scott ${ }^{3}$, Ruihua Zhang ${ }^{3}$ and Warren \\ C. Ruder ${ }^{4, *}$ \\ ${ }^{1}$ Department of Mechanical Engineering, Carnegie Mellon University, Pittsburgh, PA 15213, USA \\ 2 Department of Mechanical Engineering, Virginia Polytechnic Institute and State University, Blacksburg, VA 24061, USA \\ 3 Department of Biological Systems Engineering, Virginia Polytechnic Institute and State University, Blacksburg, VA 24061, USA \\ ${ }^{4}$ Department of Bioengineering, University of Pittsburgh, Pittsburgh, PA 15219, USA \\ * Correspondence: warrenr@pitt.edu
}

Received October 3, 2016; Revised December 12, 2016; Accepted December 20, 2016

Background: The tools of synthetic biology have enabled researchers to explore multiple scientific phenomena by directly engineering signaling pathways within living cells and artificial protocells. Here, we explored the potential for engineered living cells themselves to assemble signaling pathways for non-living protocells. This analysis serves as a preliminary investigation into a potential origin of processes that may be utilized by complex living systems. Specifically, we suggest that if living cells can be engineered to direct the assembly of genetic signaling pathways from genetic biomaterials in their environment, then insight can be gained into how naturally occurring living systems might behave similarly.

Methods: To this end, we have modeled and simulated a system consisting of engineered cells that control the assembly of DNA monomers on microparticle scaffolds. These DNA monomers encode genetic circuits, and therefore, these microparticles can then be encapsulated with minimal transcription and translation systems to direct protocell phenotype. The modeled system relies on multiple previously established synthetic systems and then links these together to demonstrate system feasibility.

Results: In this specific model, engineered cells are induced to synthesize biotin, which competes with biotinylated, circuit-encoding DNA monomers for an avidinized-microparticle scaffold. We demonstrate that multiple synthetic motifs can be controlled in this way and can be tuned by manipulating parameters such as inducer and DNA concentrations.

Conclusions: We expect that this system will provide insight into the origin of living systems as well as serve as a tool for engineering living cells that assemble complex biomaterials in their environment.

Keywords: synthetic biology; artificial cells; biotin; microparticles

\section{INTRODUCTION}

The question of how life originated is one of the most interesting unsolved mysteries in science. Current hypotheses, such as those by Szostack and colleagues [1-3], often suggest that some combination of chemical elements essential for life existed in just the right

\footnotetext{
${ }^{\dagger}$ These authors contributed equally to this work.

This article is dedicated to the Special Collection of Synthetic Biology, Aiming for Quantitative Control of Cellular Systems (Eds. Cheemeng Tan and Haiyan Liu).
}

conditions to spark a chain reaction that formed prebiotic, self-replicating material systems. Out of this "primordial stew" of components, the necessary biomaterials self-assembled. In theory, these pre-biotic cells eventually evolved into living cells. This process of material self-assembly remains critical to the performance of biomaterials and biological components today, billions of years later. Researchers in this field have sought to recreate these initial, individual self-assembly chemical reactions along the way to sparking spontaneous life in vitro $[4,5]$. In parallel, another thrust into the understanding of the minimal components necessary for life has 
focused on the creation of the minimal cell, notably by Venter and colleagues [6,7]. In this work, the smallest genome from a microbe that can be grown in culture [8], Mycoplasma genitalium, has been synthesized in vitro and transplanted into another species to successfully reboot this second species into the previous species. This minimal cell can then serve as a starting point for origin of life questions as more complex genetic circuitry is added to the cell using the tools of synthetic biology.

Rather than focusing on probing the initiation of life or the minimal components needed for life, in this study, we aimed to question if any related life-initiating or similar processes continue to occur on this planet. An initial response might suggest that, no, these processes do not continue to occur, particularly because any ecological niche sufficiently rich in nutrients and energy would be rapidly colonized by quick-evolving, existent microbes that leverage these resources faster than life can selfassemble. Yet, what if microbes themselves can direct the assembly of environmental biomaterials into artificial, protocells?

Our own previous results, along with those published by others, demonstrate the feasibility of engineering cells to direct material assembly. We previously described engineered cells that manipulate biotin-streptavidin binding to program chemical assembly on surfaces [9]. Alternately, other groups have focused on engineering cells to produce curli, an extracellular amyloid fibril, and assemble materials at multiple scales [10] using this biological building block. These materials have been shown to be useful in applications ranging from catalysis [11] to nanomaterial assembly $[12,13]$. In order to engineer materials using these precisely controlled cellular processes, researchers must have access to the unique set of cellular programming tools provided by synthetic biology. Fifteen years ago, synthetic biology was launched with reports of two synthetic gene networks $[14,15]$, the "toggle switch" and the "repressilator". Rather than open-loop control of biological processes, these systems used feedback to provide a new level of complexity in engineered cellular control of gene expression. These types of engineered gene networks, also known as synthetic gene circuits, have rapidly advanced to include control structures such as counters, timers, and logic gates [16-18]. The field has also expanded from circuits initially based on DNA-protein interactions, to include circuits based on protein-protein interactions $[19,20]$. These different networks have provided significant insight into biological phenomena. For example, the toggle switch and repressilator gave insight into the motifs required for biological memory and oscillatory behavior. This trend has since been repeatedly demonstrated as different synthetic biological circuits have provided fundamental understanding of how biological networks could support memory [14,16,21], detect sequences of events [16], and especially how biology utilizes noise and stochasticity to enable phenotypic diversity [22-28]. Similarly, by engineering living cells that direct the behavior of artificial, protocells, new insights into possible fundamental behaviors may be gained.

Engineering artificial protocells has also provided significant insight into living systems [29-31]. Moving beyond the traditional concept of a cell, artificial cellular expression systems consist of minimal environments containing DNA templates, RNA polymerase, ribosomes, ATP, and other biological molecules relevant to a particular circuit [31]. Furthermore, all components can be spatially confined, such as on a microfluidic chip [29], or within a hydrophobic oil encapsulation [32]. Robust studies have been performed to understand the engineering concepts underlying the design of these systems [32]. We extended and combined models of these welldescribed systems to explore ways in which living cells could be engineered to create artificial cells.

\section{RESULTS}

As noted, there is a significant scientific incentive to create a system that allows engineered living cells to control and command the behavior of cell-free protocells. We designed such a system (Figure 1) by leveraging three previously demonstrated laboratory components: a biotinsynthesizing engineered living cell, a streptavidin-functionalized microparticle, and an encapsulated cell-free reaction. These three components, herein referred to as modules, can be strategically linked by the transport of two key analytes: biotin and biotinylated DNA. By developing a linked, reductionist model for these three components, we explored the dynamics of how engineered living cells could alter the response of protocell modules.

Module one (Figure 1A) consists of a population of engineered living cells. We previously demonstrated how Escherichia coli (E. coli) can be genetically engineered to synthesize elevated levels of biotin when provided with a biotin precursor, desthiobiotin (DTB), and exposed to an inducer molecule, such as Isopropyl $\beta$-D-1-thiogalactopyranoside (IPTG) [9]. Biotin is a commonly used molecule in biotechnology due to the strength and versatility of the biotin-streptavidin binding event [33,34]. Additionally, biotin may be readily used to functionalize DNA monomers by incorporating biotinylated primers in a PCR amplification of DNA segments, such as those encoding for the production of green fluorescent protein (GFP). Furthermore, streptavidin is frequently bound to substrates, such as polystyrene wells or nano- and micro-particles [35]. Module two (Figure 
A



D

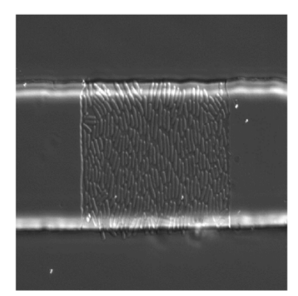

B

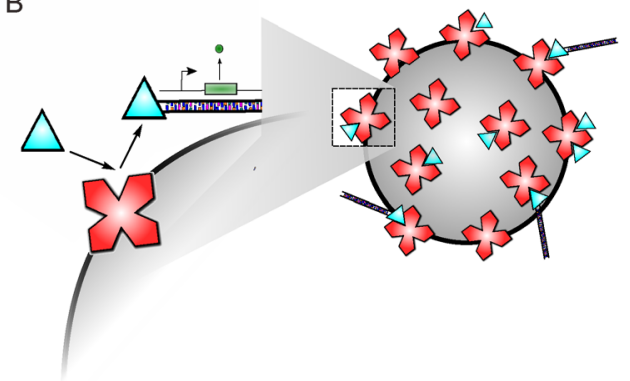

E


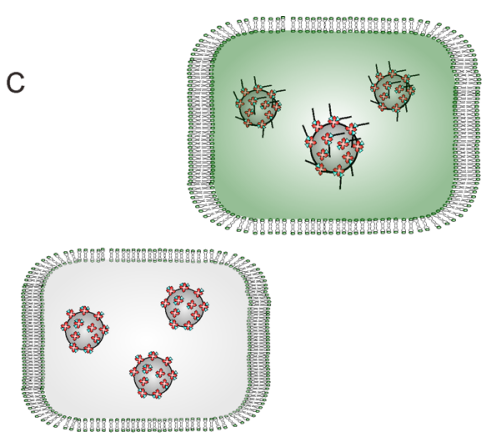

$\mathrm{F}$

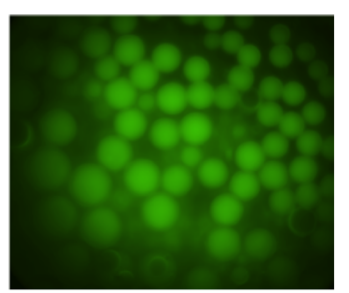

Figure 1. Linking engineered cells with cell-free systems. (A) Engineered cells synthesize biotin when exposed to a precursor molecule, desthiobiotin (DTB), and an inducer chemical such as Isopropyl $\beta$-D-1-thiogalactopyranoside (IPTG). (B) DNA encoding for GFP synthesis can be biotinylated. Streptavidin may be immobilized onto portable, micro- or nano-scale particles. When in a solution together, biotin and biotinylated DNA compete for streptavidin binding sites. (C) Particles are encapsulated with cell-free solution within a membrane to form a protocell. The protocell's transcriptional and translational behavior is governed by the concentration of DNA. (D) Genetically engineered E. coli cells are trapped in a microfluidic channel. This allows engineered cells to stay in exponential phase growth, ensuring maximum metabolic efficiency. (E) Microparticles functionalized with streptavidin bind with a biotinylated fluorophore, causing a measurable fluorescent response (E.i) and a fluorescent image (E.ii) captured using an epiflourescent microscope. (F) DNA encoding a GFP-producing cistron is encapsulated along with a TX-TL cell-free expression system within hydrofluorocarbon oil.

1B) exploits this technology by allowing cell-produced biotin and GFP-encoding, biotinylated DNA to compete for binding sites on streptavidin-functionalized microparticles.

Module three encapsulates these particles along with a cell-free TX-TL reaction to form a protocell (Figure 1C). Cell-free systems are widely used to explore biological phenomena such as gene network dynamics [36] and molecular assembly [37]. The intensity of a cell-free protein synthesis response is governed by the concentration of DNA available within the cell-free encapsulation. As such, in module three the intensity of a green fluorescent signal is proportional to the concentration of GFP-encoding, biotinylated DNA bound to the microparticles.

Taken together, these three modules provide a framework that enables programmable cells to control the response of cell-free protocells. In addition to engineering a strain of biotin producing cells, researchers have experimentally demonstrated a number of enabling technologies for all three modules. For instance, microfluidic channels (Figure 1D) may be used to contain, and provide nutrients to, populations of engineered cells [38]. These microfluidic chips allow ease of imaging [39] while keeping the contained cells in a prolonged exponential growth phase [38]. Additionally, streptavidin functionalized microparticles can bind to a biotinylated fluorophore eliciting a measurable fluorescent response (Figure 1E). Finally, GFP-encoding DNA may be encapsulated within a droplet containing TX-TL cell-free extract [40] to elicit a strong fluorescent response (Figure 1F).

Additionally, all three modules may be modeled in a reductionist fashion from kinetic principles and simulated using established numerical methods. By providing a mathematical underpinning for our system, these models enable predictive testing and insight, allowing us to better understand the governing dynamics of the system's cellprotocell interaction.

Module one leverages the tools of synthetic biology to genetically program the behavior of cells. Many well- 
A



B

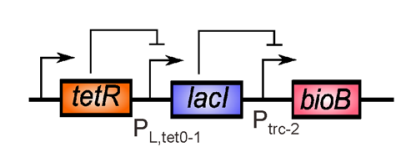

C

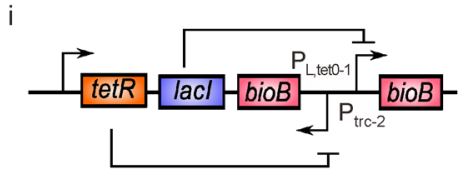

D

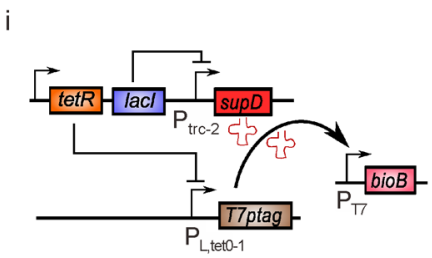

E

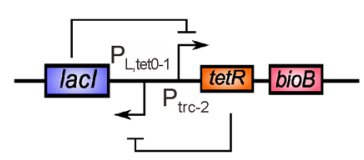

ii

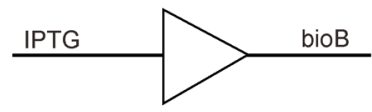

ii



ii



i

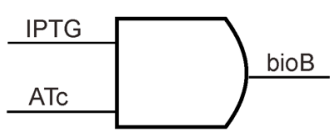

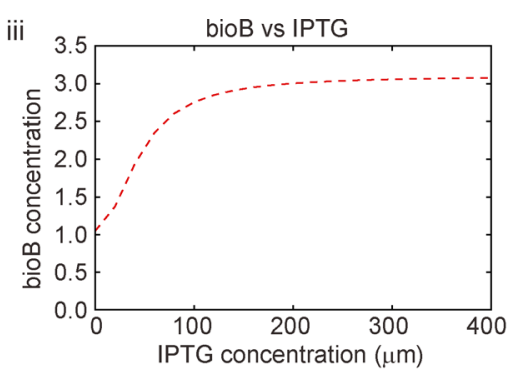

iii



bioB concentration


ii

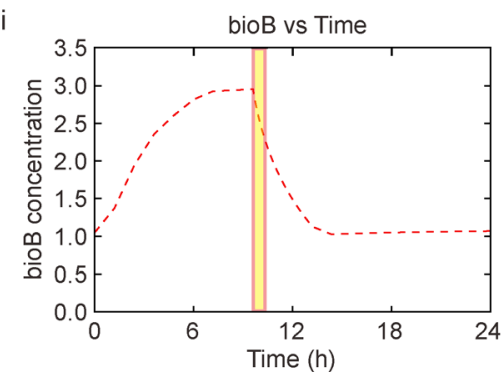

Figure 2. Programming engineered cells. Synthetic gene regulatory networks are used to control the behavior of engineered cells. (A) The inducer/driver gene regulatory network (A.i) consists of a lacl-repressed promoter site driving the transcription of bioB, a gene encoding for biotin synthase. Then, when introduced, IPTG binds to lacl, inhibiting lacl's ability to repress the promoter site. This induces the transcription of bioB. An electrical logic abstraction is shown (A.ii) to illustrate this system. Existing predictive, continuous models allow us to simulate this circuit's behavior (A.iii). Similar representations are shown for a genetic inverter (B), a genetic OR-gate (C), a genetic AND-gate (D), and a genetic toggle switch (E). 
established genetic motifs (Figure 2), inspired by electronic logic, have been synthesized and transformed into $E$. coli as well as other organisms. A simple gene regulatory network is the analogue inducer, or driver, circuit (Figure 2A.i). Genes encoding for the repressor protein lacI are downstream from a constitutive promoter site [41]. In the absence of a chemical inducer, lacI binds to a $\mathrm{P}_{\text {trc-2 }}$ promoter site, inhibiting the transcription of downstream sequences. However, when the inducer molecule IPTG is introduced to the cells, lacI disassociates from the DNA strand and binds with the available IPTG. This derepression of the $\mathrm{P}_{\text {trc-2 }}$ promoter site allows for transcription of downstream genes, such as bioB, a gene encoding for the production of the enzyme biotin synthase [42]. An electrical circuit analogy (Figure 2A.ii) depicts the dynamics of this system, whereby additional IPTG causes an increase in biotin synthase production. This behavior may be simulated (Figure 2A.iii) using commonly employed genetic models further described in the methods section [43-45].

Other well-established gene networks can be designed, modeled, and simulated in a similar manner. A simple modification to the inducer circuit is an inverter circuit (Figure 2B.i) in which an inducing chemical, this time anyhydrotetracycline (ATc), attenuates the production of biotin synthase. This behavior is accomplished by placing the lacI gene downstream from a $\mathrm{P}_{\mathrm{L} \text {,tetO-1 }}$ promoter site, and allowing the repressor protein, tetR, to be produced constitutively. In the absence of ATc, tetR represses the production of lacI, allowing for robust bioB transcription. However, when ATc is introduced to the system, it binds to the tetR repressor, in turn upregulating lacI synthesis. This has the effect of repressing the $\mathrm{P}_{\text {trc-2 }}$ promoter site, decreasing bio $B$ transcription and biotin synthase translation. The electrical circuit analogy (Figure 2B.ii) and simulated response profile (Figure 2B.iii) confirm this behavior.

Similarly, an OR-gate, in which the presence of ATc or IPTG causes elevated levels of biotin synthase production, may be engineered (Figure 2C). This logic gate is accomplished by placing $b i o B$ downstream from both a $\mathrm{P}_{\text {trc-2 }}$ and a $\mathrm{P}_{\mathrm{L}, \text { tetO-1 }}$ promoter sites. Another commonly used genetic motif is the AND-gate (Figure 2D), in which only the presence of both ATc and IPTG will cause elevated levels of biotin synthase production [46]. This is accomplished by controlling the production of viral $\mathrm{T} 7$ polymerase, which in turn binds to a $\mathrm{T} 7$ promoter site driving $b i o B$. With only IPTG present, this system creates short non-coding hairpin RNA sequences. When only ATc is introduced, the AND-gate transcribes an RNA encoding for $\mathrm{T} 7$ that has been compromised with internal stop-codons. However, when both ATc and IPTG are introduced to the cell, the hairpin RNA sequences bind to the superfluous stop codons on the T7 polymerase RNA sequence. This allows for effective translation of the T7 polymerase, which in turn activates biotin synthase production. Finally, we can model a toggle-switch (Figure $2 \mathrm{E}$ ), in which sustained periods of activation or repression may be triggered by a transient pulse of either ATc or IPTG $[47,48]$. This behavior is accomplished by designing the lacI and tetR genes to be mutually repressed. Simulated behaviors for the OR-gate, AND-gate, and toggle switch (Figure 2C, 2D, and 2E) provide us with predictable insight into the dynamic behavior of these gene regulatory networks.

However, biotin synthesis is a function of both bioB regulation and the cell's access to available DTB. Previously, we developed equations for a response profile by fitting basic enzyme kinetic equations with experimental results $[9,49]$. Taking the resulting equations, and coupling them with the biotin synthase response profiles developed in Figure 2, we can model and simulate biotin synthesis as a function of inducer molecules and available DTB. After simulating an inverter circuit, the biotin response profile (Figure 3) depicts a decrease of biotin production as more ATc is added to the system while showing that an increase in DTB causes an increase in biotin production. This is the expected response; the inverter circuit attenuates $\operatorname{bio} B$ transcription, and thereby biotin formation, as more ATc is introduced to the cells. Similar response profiles may be simulated for all of the gene networks presented in Figure 2, allowing us to rationally design gene networks for a predictable biotin response.



Figure 3. Controllable biotin synthesis from engineered cells. Cells containing an inverter circuit (Figure 2B) were simulated. The resulting biotin produced was plotted as a function of ATc and DTB. 
Module two exploits the versatility of the biotinstreptavidin bond. By establishing a competitive binding interaction between biotin produced by the engineered cells, and biotinylated DNA, we can predictably model the percent of streptavidin sites occupied by biotinylated DNA for an experimentally relevant range of biotin concentrations. This model (Figure 4A) employs a fourparameter logistic response profile, fit with previously published constants and experimental data $[9,50]$. Furthermore, it allows us to explore how altering the concentration of biotinylated DNA (orange, yellow, blue, black) can affect the response of the initial (green) curve.

Coupling this competitive binding response function with information about the streptavidin-functionalized microparticles allows us to calculate the concentration of biotinylated DNA bound to the particles. A twodimensional response profile (Figure 4B) may be developed relating the concentration of DNA per-weight of particle to the concentration of biotin and the radius of the particles. Note that smaller particles and lower concentration of biotin cause a larger concentration of bound, biotinylated DNA. This profile enables effective experimental parameter tuning by predictively altering the size of particles and dilution of biotin.

Module three is an encapsulation composed of DNA (bound to microparticles) and cell-free extract. This module allows us to monitor the behavior of a cell-free system by examining an encapsulated droplet and analyzing the fluorescent response [51]. By modifying the concentration of DNA encoding for GFP within a cellfree system, previous studies have developed a reliable

A

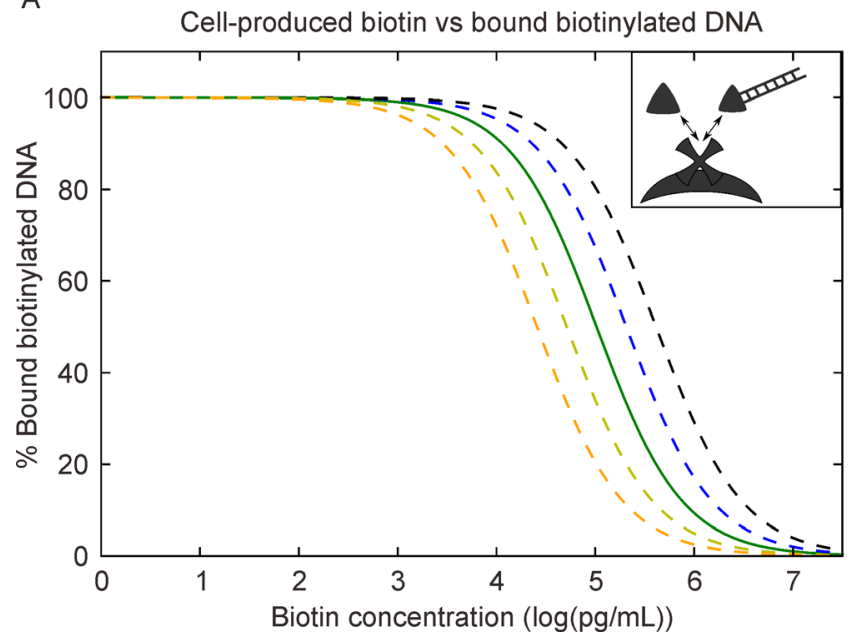

framework for modeling cell-free response as a function of DNA concentration [52]. Here, we simulate the temporal response for three concentrations of DNA (Figure 5A) and then plot the maximum GFP expression (Figure 5B) as a function of initial DNA concentration. We find that there is an asymptotically stable response profile relating the DNA concentration to the fluorescent intensity. This curve suggests that reactions employing low concentrations $(0.0-20.0 \mathrm{ng} / \mathrm{uL})$ of DNA will result in larger fluorescent intensity charges, a favorable experimental condition.

The models for all three modules may be linked by strategic chemical transport. The biotin produced by module one directly affects the competitive binding in module two which in turn directly affects the concentration of DNA encapsulated within the cell-free system. By specifying experimental conditions, such as DTB concentration and particle sphere radius, the linked modules model allow us to simulate cell-free fluorescent response as a function of the inducer concentration exposed to the engineered cells (Figure 6).

First, we simulate a biotin response profile for a population of engineered cells (Figure 6A). We chose to simulate the inducer/driver circuit for illustrative purposes. By specifying a concentration of DTB, we can plot the concentration of biotin produced as a function of IPTG. Passing this biotin through module two, we can calculate the percent of particle-bound biotinylated DNA (Figure 6C) as a function of IPTG. Upon selecting a particle radius, we can calculate the concentration of DNA bound to the particles, which may be used to

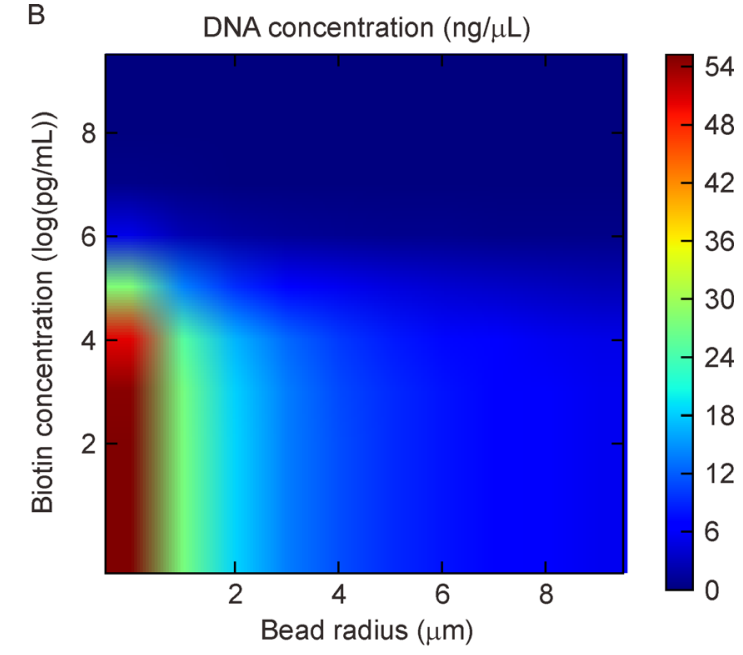

Figure 4. Functionalized microparticles interact with cell-produced biotin. Cell-produced biotin and biotinylated DNA compete for streptavidin binding sites. (A) The competitive binding dynamics are modeled and tuned with previous experimental results (green). Shifts in the dynamic range occur by altering the concentration of biotinylated DNA within the system (yellow, orange, blue, black). (B) Total bead-bound DNA concentration per weight of beads may be calculated as a function of cell-produced biotin and the radius of the bead selected. 



Figure 5. Fluorescent response of a cell-free system. (A) The temporal dynamics of a cell-free systems may be modeled as a set of five ordinary differential equations, and simulated. (B) The maximum/steadystate levels of GFP produced by a cell-free system may be plotted as a function of the DNA concentration.

simulate a cell-free response (Figure 6D). By indexing the simulated DNA concentrations with the IPTG concentrations, we can plot a function relating the concentration of IPTG given to the engineered cells with the GFP production within the cell-free system (Figure 6E). In such a manner, we can simulate and predict how engineered cells can control the behavior of cell-free encapsulations and protocells.

\section{DISCUSSION}

As previously discussed, the key hypothesis we explored is the possibility that living cells can be engineered to direct the assembly of genetic components required for protocells, potentially revealing a possible complex biological behavior motif. Our work here explores one component of this, the assembly of DNA circuitry on a programmable material scaffold and opens the door to the possibility that cells could assemble complex systems from materials scavenged from their environment. Moreover, microbes have frequent access to these building blocks of life as cell death ends in lysis, and the result is a microbial environment awash in functional macromolecules. Finally, one might ask if microbes would benefit from creating a cell-like system in their environment? Could they create such a material structure? In fact, most microbes in nature exist as part of multispecies consortia, and these consortia often collaboratively engage in extracellular material assembly in the process of biofilm formation. The fitness advantages conferred to a biofilmencapsulated consortium are significant (e.g., dental tartar conveys protection from the abrasive cleaners). By assembling cell-like systems from components in their environment, a mixed-species consortium could abrogate the need for any one member to evolve all the components of a new genetic pathway. Thus, microbes have both the materials and the evolutionary fitness motivation to direct the assembly of artificial cells.

In the work here, we showed how a single population and cell strain could be developed to control assembly. However, there is also the promising potential to use an engineered consortium [53-55] for similar molecular assembly. These complementary synthetic systems have already been introduced. As a first step, programmable material surfaces would be engineered that serve to anchor individual components of engineered genetic circuits. Living cells would then be engineered to program the self-assembly of circuit components on these material surfaces similar to the ways that have been demonstrated. The assembled genetic circuits will then be assayed using a cell-free, gene expression system. As a second step, given that we encoded most of the information exchange between modules using the transport of biological molecules, a microfluidic system enabling the rapid, controlled assembly of biological materials within liposomes could be developed as a critical enabling technology. Both the engineered living cells and the microfluidic assembly system could be combined in the development of a unique ecological consortium consisting of living cells and non-living, cellinspired material systems. In these experiments, a mixture of living cells and the artificial cells they assemble would be engineered to be co-dependent members of an engineered consortium [56]. The living components of these systems will only propagate if they continue to participate in the assembly of the non-living artificial cells.

By creating these types of engineered microbial and artificial cell consortia, we believe that a range of problems could be explored where diverse synthetic biological systems would be useful, ranging from drug 

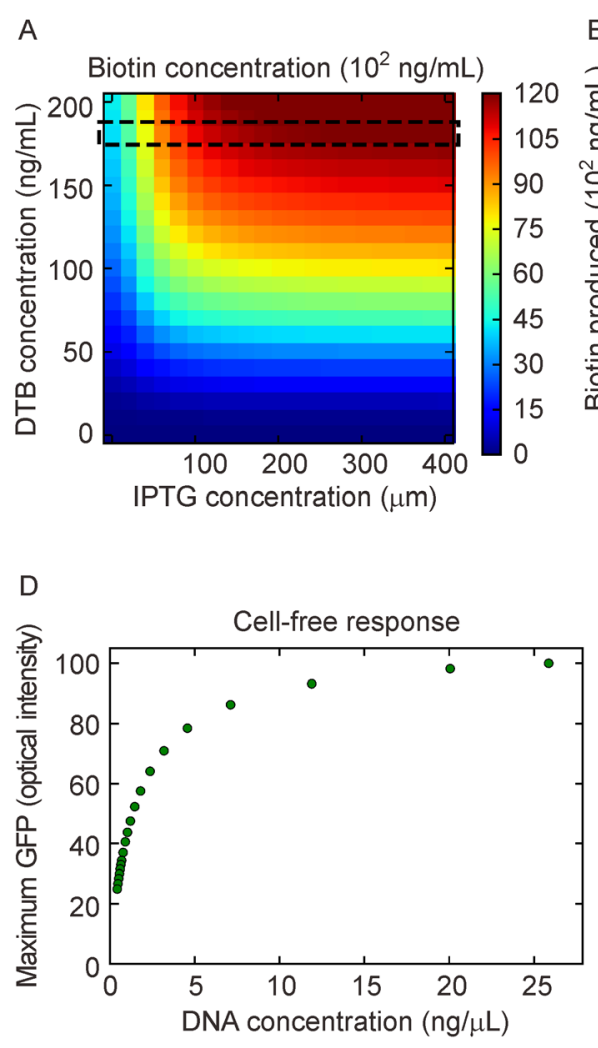
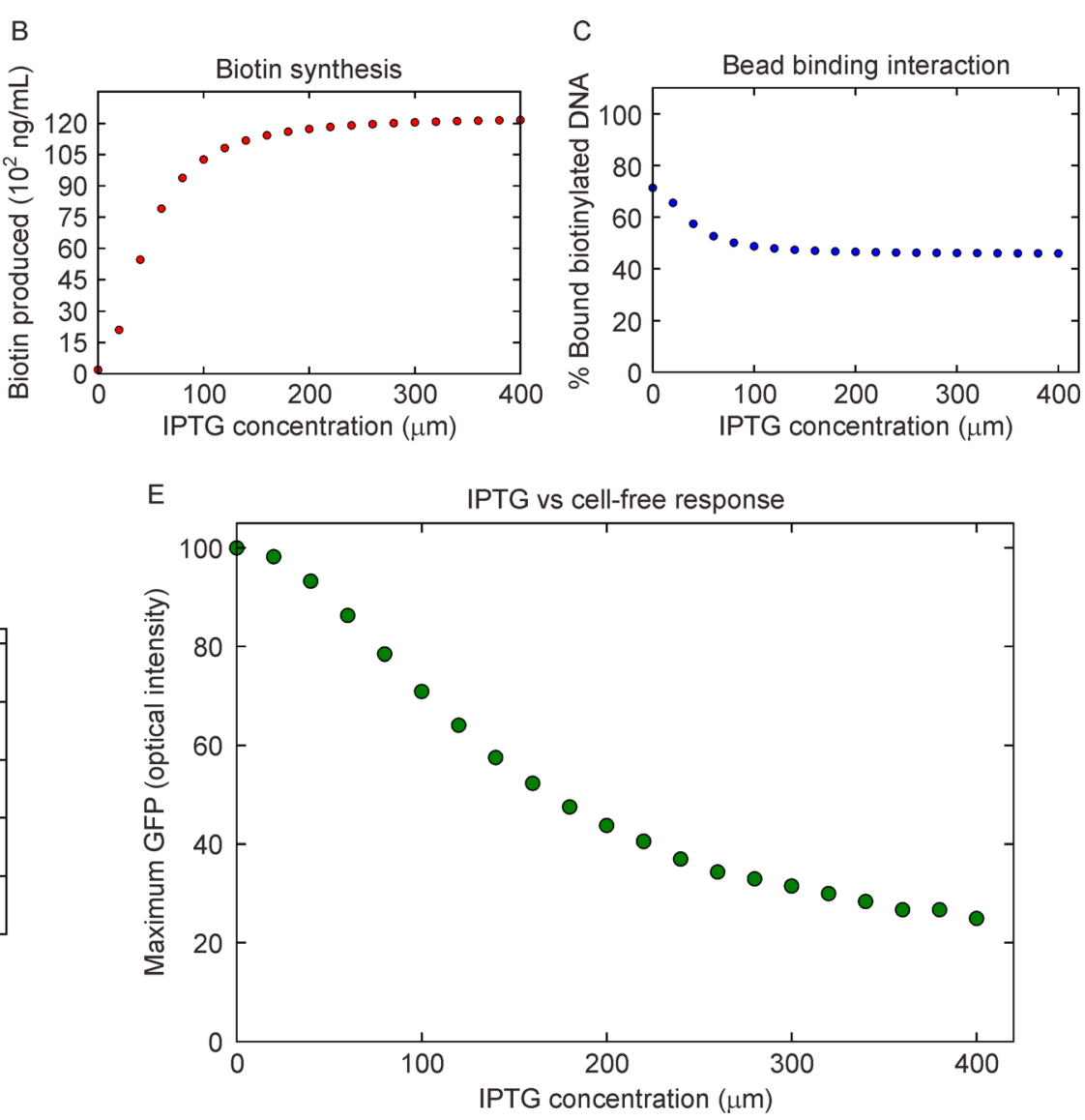

Figure 6. Engineered cells control the dynamics of cell-free protocells. (A) Biotin synthesis response profiles for cells engineered to contain an inducer circuit. (B) Biotin synthesis response profile as a function of the inducer molecule, IPTG for a constant DTB value. (C) Bound, biotinylated DNA as a function of the IPTG introduced to the cells. (D) The maximum GFP response produced by the concentrations of DNA calculated from (D) with a constant bead radius. (E) By mapping (D) with (C), we plot the cell-free GFP produced as a function of the IPTG input to the engineered cells.

delivery and materials science to the fundamental understanding of the origin of life.

\section{MATERIALS AND METHODS}

\section{Experimental methods}

Preparation of PDMS chip

Master molds were fabricated in the Virginia Tech Micro \& Nano Fabrication Laboratory (MICRON). Photomasks were drawn in AutoCAD (San Rafael, CA), printed onto transparency film by CAD/Art Services, Inc. (Output City, Poway, CA), and mounted onto glass plates (McMaster-Carr, Los Angeles, CA). The first layer of the master mold was created by spin coating a layer of SU-8 2000 negative photoresist (MicroChem Corp., Newton, MA) onto a clean silicon wafer to a height of $0.96 \mu \mathrm{m}$ using a WS-650-8B programmable spinner (Laurell Technologies Corp., PA). Then the photoresist was exposed to ultraviolet light (UV) using a MA/BA6 UV contact mask aligner (MicroTec, Garching, Germany). After UV exposure, the wafer was rinsed using a developer (MicroChem Corp., Newton, MA) to remove residual, unexposed photoresist. For the second layer, SU-8 2010 negative photoresist was spin coated on top of the first layer (again using the WS-650-8B spinner) and brought to a total height of $10 \mu \mathrm{m}$. The wafer then underwent another round of UV exposure, using the MA/ BA6 UV contact mask aligner, followed by another round of developer rinsing.

Replica molds were then created from the master mold. PDMS/Sylgard 184 (Dow Corning, Midland, MI) was first mixed at a 10:1 ratio with an elastomer base curing agent. This mix was poured on top of the wafer, and allowed to degas in a vacuum desiccator for 1 hour. The mold was then cured in an oven at $90^{\circ} \mathrm{C}$ for 2 hours. The PDMS chips were then removed from the oven, and holes were punched into the locations designed for liquid ports I/O ports. Chips were then cleaned using filtered DI water, 
and strongly bound to clean coverslips (Thermo Fisher Scientific, Waltham, MA). The coverslip and PDMS chip were bound by exposing both surfaces to oxygen plasma for 1 minute in the PDC-32G plasma cleaner (Harrick Scientific, Ithaca, NY) and placing the exposed surfaces together.

Once bound, chips were wetted by flowing a $0.05 \%$ Tween in DI water solution through the chip for 1 hour. Cells were loaded into the trapping channels of the microfluidic device by directing flow in the "forward" direction from the cell port to the waste port. Upon trapping a few cells in each region, the flow was reversed and slowed to a rate of $1 \mathrm{~mL} /$ day. This allowed a steady supply of nutrients to the entrapped cells. Cells were imaged while being incubated at room temperature (RT) for 6-10 hours. The chip's design allowed cells to grow continuously in their exponential phase.

Images were acquired using an inverted epifluorescent microscope (Ti-E, Nikon Instruments Inc.). Throughout long-term imaging, the PDMS chip was maintained at $22^{\circ} \mathrm{C}$. DIC images were taken every 5-10 minutes, and optical focus was maintained automatically using the Nikon Elements software package.

\section{Biotinylated fluorophore with streptavidin microbeads}

A green, biotinylated fluorophore was experimentally bound to streptavidin coated, red fluorescent beads to test the binding interaction of biotin and streptavidin. To create the solution mixture, $10 \mu \mathrm{L}$ of $0.1 \% \mathrm{w} / \mathrm{v}$ streptavidin coated microbeads (Spherotech ${ }^{\circledR}$, FSVM-2058-2) were mixed with $10 \mu \mathrm{L}$ of $0.01 \mathrm{M}$ green biotinylated fluorophore, Atto 520-Biotin (Sigma Aldrich, 01632), and incubated for 1 hour at RT in a phosphate buffer, $\mathrm{pH}$ 7.2. The beads were then washed 6 times with PBS using a 6 tube magnetic separator (NEB, S1506S). The final bead solution was suspended in $50 \mathrm{~mL}$ PBS. Fluorescent intensity of the beads was used to indicate the strength of the biotin binding event. To image, a $2 \mu \mathrm{L}$ droplet of solution was placed on a clean coverslip. Imaging was completed using a Nikon eclipse $\mathrm{Ti}$ with a mounted Andor ${ }^{\circledR}$ Zyla scientific CMOS (sCMOS) camera (Ti-E, Nikon Instruments Inc.)

\section{Cell-free encapsulations}

The TX-TL cell-free expression protocol from the Noireaux lab[40] was used to make the components of the cell-free reaction. First, crude cell extract was made using frozen stocks of Rosetta ${ }^{\mathrm{TM}}$ (DE3) E. coli BL21 cells, genotype $F-$ ompT $h s d S B(r B-m B-)$ gal dcm (DE3) pLysSRARE (CamR) grown in $2 \times \mathrm{YT}+\mathrm{P}$ and chloramphenicol $(\mathrm{Cm})$ media. Cultures were grown for 8 hours at $37^{\circ} \mathrm{C}$ with agitation at $220 \mathrm{rpm}$. The cells were then by subcultured and further incubated under the same conditions for an additional 4 hours $\left(\mathrm{OD}_{600}=1.5-2.0\right)$. The cells were then pelleted through centrifugation and washed using S30A buffer. After centrifuging, the pellet was weighed. S30A buffer and $0.1 \mathrm{~mm}$ glass beads were then mixed with the pellet through vigorous vortexing. The bead-cell mixture was aliquoted into bead beating tubes and cells were lysed by bead beating each tube twice at $46 \mathrm{rpm}$ for 30 seconds. To remove the beads, micro-chromatography columns were pressed to the ends of open tubes after beating and were centrifuged. Of the eluted sample, the supernatant was collected and incubated for $80 \mathrm{~min}$ at $37^{\circ} \mathrm{C}$ with $220 \mathrm{rpm}$ agitation to further digest nucleic acids with endogenous exonucleases. The samples were spun down again and the supernatant was collected and dialyzed using 10k MWCO dialysis cassettes submerged in S30B buffer with stirring for 3 hours at $4{ }^{\circ} \mathrm{C}$. The processed extract was spun down one last time and the supernatant was divided into $30 \mu \mathrm{L}$ aliquots, flash-frozen, and stored at $-80^{\circ} \mathrm{C}$. Protein concentration $(30 \mathrm{mg} / \mathrm{mL})$ of the extract was measured using a Bradford assay.

Amino acid and energy solutions were combined into buffer solutions and calibrated according to previously published protocols [40]. The plasmid pZE21-GFP $\left(\mathrm{P}_{\mathrm{L}, \text { tetO }}\right.$ driving green fluorescent protein (GFP) expression) was used to visualize protein production within the cell-free system. Optimal concentrations of Mg-glutamate, K-glutamate, and dithiothreitol (DTT) were determined experimentally, and found to be $6 \mathrm{mM}, 140 \mathrm{mM}$, and $1 \mathrm{mM}$, respectively. In addition, each experimental reaction also consisted of $1.5 \mathrm{mM}$ amino acids, $1 \times$ energy solution, 2\% PEG-8000, 33\% extract, and DNA. The concentration of DNA may be adjusted by calculating the molar concentration of the stock and varying the ratio of DI water to DNA during reaction construction.

Reactions were incubated at $29^{\circ} \mathrm{C}$ in a thermocycler for 8 hours. After incubation, reactions were imaged by encapsulating cell-free reactions within oil-immersion droplets by adding $15 \mu \mathrm{L}$ Triton ${ }^{\circledR}$ X-100 (CAS 9002-931) to each $10 \mu \mathrm{L}$ reaction and quickly flicking the tube. 2 $\mu \mathrm{L}$ of the cell-free encapsulation mixture was pipetted onto a cover slip and immediately observed and imaged using a Nikon eclipse Ti with a mounted Andor ${ }^{\circledR}$ Zyla scientific CMOS (sCMOS) camera.

\section{Modeling methods}

Module one: the engineered cell

Module one consists of a population of E. coli cells engineered to contain gene networks capable of synthesizing biotin synthase, which in turn enables the cells to produce biotin. In previous publications, we detail how a 
continuous model, depicting the system's dynamic behavior, may be developed for complex regulatory gene networks [45,57] as well as the biotin synthesizing processes [9]. This approach relies upon modeling four key subprocesses:

i) Inducer mass balance and binding kinetics

ii) Transcription of mRNA from regulatory gene components

iii) Translation of proteins, including reporters, repressors, and biotin synthase

iv) Biotin metabolism

The first subprocess is modeled by establishing three mass balance equations for a given inducer. These equations relate the concentrations of internal inducers, $\mathrm{I}_{\text {int }}$, repressor proteins, TP, and bound inducer-repressor complexes TP: $\mathrm{I}_{\text {int }}$ within a cell (Equations (1)-(3)). In addition to a simplified membrane flux term, these mass balances use first-order binding kinetics to relate the relative concentrations of bound and unbound internal inducer and repressor protein complexes. A full derivation may be found in previously published literature by the authors [57].

$$
\begin{gathered}
\frac{\mathrm{d}\left[\mathrm{I}_{\text {int }}\right]}{\mathrm{dt}}=\mu\left(\left[\mathrm{I}_{\mathrm{ex}}\right]-\left[\mathrm{I}_{\mathrm{int}}\right]\right)-\mathrm{K}_{\mathrm{a}}\left[\mathrm{I}_{\mathrm{int}}\right] \times[\mathrm{TP}] \\
+\mathrm{K}_{\mathrm{d}}\left[\mathrm{TP}: \mathrm{I}_{\mathrm{int}}\right]-\delta_{1} \times\left[\mathrm{I}_{\mathrm{int}}\right] . \\
\frac{\mathrm{d}[\mathrm{TP}]}{\mathrm{dt}}=-\mathrm{K}_{\mathrm{a}}\left[\mathrm{I}_{\text {int }}\right] \times[\mathrm{TP}]+\mathrm{K}_{\mathrm{d}}\left[\mathrm{TP}: \mathrm{I}_{\mathrm{int}}\right]+g[\mathrm{TP}] . \\
\frac{\mathrm{d}\left[\mathrm{TP}: \mathrm{I}_{\text {int }}\right]}{\mathrm{dt}}=\mathrm{K}_{\mathrm{a}}\left[\mathrm{I}_{\mathrm{int}}\right] \times[\mathrm{TP}]-\mathrm{K}_{\mathrm{d}}\left[\mathrm{TP}: \mathrm{I}_{\text {int }}\right] \\
-\delta_{3} \times\left[\mathrm{TP}: \mathrm{I}_{\text {int }}\right] .
\end{gathered}
$$

Equation (1) describes the time rate of change of the internal inducer concentration, $\mathrm{I}_{\text {int }}$, as a function of four terms. The first term on the right hand side is a membrane transport term used to describe the mass flux of an inducer, such as IPTG, across the cell membrane. The second term is a kinetic association term that relates the internal concentration of an inducer, $\mathrm{I}_{\mathrm{int}}$, with the internal concentration of a transcription regulation protein, TP, and a kinetic association constant, $\mathrm{K}_{\mathrm{a}}$. The third term describes the disassociation of the repressor proteininducer molecule complex, described by a disassociation constant, $\mathrm{K}_{\mathrm{d}}$, and the concentration of the bound complex, TP: $I_{\text {int }}$. Finally, the fourth term describes the decay of the internal inducer. It should be noted that for some inducers, such as lactose or arabinose, this fourth term is a function of natural cell metabolism.

Equation (2) describes the time rate of change of unbound transcription regulation protein available within the cell. The terms on the right hand side are analogous to those described in Equation (1). However, there is an additional term describing the rate of regulation protein generated by the cell through the process of translation,
$g[\mathrm{TP}]$. Note that this translation process is described for a generic protein by Equation (5), and that transcription factor proteins such as lacI or tetR, denoted as TP, are specific instance of a generic protein, denoted by $\mathrm{P}$.

Finally, Equation (3) describes the time rate of change of the bound inducer-transcription factor protein complex with terms analogous to those described in Equation (2) and Equation (3). Additionally, there is a decay term that is composed of a decay constant and the concentration of bound complex. It should be emphasized that this modeling approach was used for its simplicity and versatility to describe a wide array of inducer-repression complexes with the dynamics of the system emphasized.

The second sub-process, mRNA transcription, is modeled as a Hill-like function (Equation (4)) in order to capture the dynamics of the gene network without adding excess complexity. This approximation is justified by considering our system to contain a population of E. coli cells rather than relying on single-cell dynamics. The large population, coupled with a high copy count plasmid should mitigate significant statistical outliers. The equation relates the rate of mRNA synthesis with the concentration of the relevant reporter protein, the transcription leak, and the decay of mRNA.

$$
\begin{aligned}
\frac{\mathrm{d}[\mathrm{mRNA}]}{\mathrm{dt}}= & \mathrm{V}_{1 \max }\left(\frac{1}{1+\left(\frac{[\mathrm{TP}]}{\mathrm{K}_{\mathrm{b}}}\right)^{\mathrm{H}}}\right) \\
& +\mathrm{V}_{\text {lleak }}-\delta_{\mathrm{mRNA}} \times[\mathrm{mRNA}] .
\end{aligned}
$$

Equation (4) describes the time rate of change of a generic mRNA concentration, mRNA, using three terms. The first term on the right hand side is a Hill-like function containing the maximum inducible rate of mRNA transcription, $\mathrm{V}_{1 \max }$, the concentration of repressor transcription factor proteins, TP, a kinetic constant, $\mathrm{K}_{\mathrm{b}}$, and a Hill coefficient, $\mathrm{H}$. The second term is a constant describing the amount of transcriptional leak within the cell, $\mathrm{V}_{\text {lleak, }}$, and the third term describes the decay of mRNA within the cell by relating a decay constant, $\delta_{\mathrm{mRNA}}$, and the mRNA concentration, mRNA.

Protein translation, the third subprocess, is modeled by relating the rate of protein synthesis with the concentration of available mRNA, ribosome binding site strength (RBS), and a decay factor (Equation (5)). This equation is derived from Michaelis-Menten kinetics and is often used to model protein expression [44] when studying gene regulatory networks. Additionally, note that Equation (5) is adapted and utilized within Equation (2) as g[TP], the generation of a transcription factor protein. This inclusion allows us to account for transcription factor generation in the context of the binding kinetics described within Equation (2). Additionally, the production of the protein biotin synthase can be recognized as a specific instance of 
a protein generated by the translation process described within Equation (5).

$$
\frac{\mathrm{d}[\mathrm{P}]}{\mathrm{dt}}=\mathrm{V}_{2 \max } \times \mathrm{RBS} \times \frac{[\mathrm{mRNA}]^{\mathrm{m}}}{\mathrm{K}_{2}{ }^{\mathrm{m}}+[\mathrm{mRNA}]^{\mathrm{m}}}-\delta_{\mathrm{p}} \times[\mathrm{P}] .
$$

With Equation (5), we model the time rate of change for a generic protein, $\mathrm{P}$, as a function of two terms. The first term describes the formation of the protein as the product of the maximum rate of translation, $\mathrm{V}_{2 \max }$, a constant describing ribosome binding strength, and a Hill function relating the concentration of mRNA, with an activation coefficient, $\mathrm{K}_{2}$, and a Hill coefficient, $\mathrm{m}$. The second term describes the rate of decay for a specific protein, relating a decay constant, $\delta_{p}$ and the intercellular protein concentration for a generic protein.

Finally, the fourth sub-process describes the biotin formation by the cell. A simple model was developed for this process, inspired by Michaelis-Menten formalism, relating the concentration of available biotin synthase and DTB with the rate of biotin produced by the cell.

$$
\begin{aligned}
\frac{\mathrm{d}[\text { biotin }]}{\mathrm{dt}}= & \mathrm{V}_{3 \max } \times[\text { bioB }] \times \frac{[\mathrm{DTB}]^{\mathrm{q}}}{\mathrm{K}_{3}{ }^{\mathrm{q}}+[\mathrm{DTB}]^{\mathrm{q}}} \\
& -\delta_{\text {biotin }} \times[\text { biotin }] .
\end{aligned}
$$

With Equation (6), we model the time rate of change of biotin concentration as the sum of a Hill function describing the rate of biotin synthesis and a decay term. The Hill function contains parameters describing the max rate of formation, $\mathrm{V}_{3 \max }$, an activation coefficient, $\mathrm{K}_{3}$, and a Hill coefficient, q. Crucially, the rate of biotin formation is governed by the concentrations of both biotin synthase, bioB, and DTB. Parameters for this equation were bounded and fit using our previously published experimental data[9]. It should be noted that the rate of biotin decay is orders of magnitude slower than the rate of biotin formation, and as such the decay term can be largely ignored.

In our simulation, parameter values were taken from results previously published by other groups $[33,52,58$ 60], as well as our own previously published experimental results [9]. As a characteristic example, the parameter values used for the inducer circuit shown in Figure 6 were as follows: the IPTG relevant membrane permeability constant [58], $\mu=5.0 \times 10^{-2} \mathrm{~m}^{-1}$, the kinetic association constant [59], $\mathrm{K}_{\mathrm{a}}=1.0 \times 10^{3} \mathrm{~m}^{-1}$, the kinetic disassociation constant, $\mathrm{K}_{\mathrm{d}}=1.0 \times 10^{-2} \mathrm{~m}^{-1}$, and timescale negligible decay constants [60], $\delta_{1}=\delta_{3}=0$.

Equations (4)-(6) were fit using previously published data describing a planktonic culture of $3 \mathrm{~mL}$ of $E$. coli held at $37^{\circ} \mathrm{C}$ for 24 hours of growth [9]. Under these conditions, the transcription parameters are as follows: $\mathrm{V}_{1 \text { max }}=3.0 \mathrm{nM} \times \mathrm{m}^{-1}, \mathrm{~V}_{\text {1leak }}=4.15 \mathrm{nM} \times \mathrm{m}^{-1}, \delta_{\text {mRNA }}=$
$10.0 \mathrm{~m}^{-1}, \mathrm{~K}_{\mathrm{b}}=1.0 \times 10^{2} \mathrm{nM}$, and $\mathrm{m}=2$. Additionally, for the translation of biotin synthase, the parameters are: $\mathrm{V}_{2 \max }=6.2 \times 10^{2} \mathrm{nM} \times \mathrm{m}^{-1}, \mathrm{~K}_{2}=10.0 \mathrm{nM}, \mathrm{RBS}=1.0$, $\mathrm{m}=2$, and $\delta_{\mathrm{P}}=1.0 \mathrm{~m}^{-1}$. Finally, for the process of biotin synthesis, the parameters values were as follows: $\mathrm{V}_{3 \max }=$ $6.2 \times 10^{2} \mathrm{ng} \times \mathrm{mL}^{-1} \times \mathrm{m}^{-1}, \mathrm{~K}_{3}=1.0 \times 10^{2} \mathrm{nM}, \mathrm{q}=2$, and $\delta_{\text {biotin }} \cong 0$.

Module two: streptavidin functionalized microbeads

Biotin-streptavidin interactions have been widely studied; here we were able to use a well-fit model from previously published results and apply it to our microbead system. Using this model (Equation (7)) we can describe the competitive binding interaction between free biotin and biotinylated DNA for streptavidin sites. Modifying the concentration of biotinylated DNA introduced to the system allows us to shift the dynamic range of the curve higher or lower. A full derivation, as well as the experimental data to which the parameters were fit, was previously published by the authors [9].

$$
\begin{aligned}
\% \mathrm{SA}_{\mathrm{DNA}}= & D \\
& +\frac{\mathrm{K}_{4} \times\left[\operatorname{Biotin}_{\mathrm{DNA}}\right]-\mathrm{D}}{1+\mathrm{K}_{4} \times\left[\text { Biotin }_{\mathrm{DNA}}\right]+\mathrm{K}_{5} \times \text { Biotin }_{\text {Cells }}} .
\end{aligned}
$$

With Equation (7), we model the percent of streptavidin sites bound to biotinylated DNA $\left(\% \mathrm{SA}_{\mathrm{DNA}}\right)$ as a function of a background parameter, D, two association constants, $\mathrm{K}_{4}$ and $\mathrm{K}_{5}$, the concentration of biotinylated DNA, Biotin $_{\mathrm{DNA}}$ and the concentration of cell-produced biotin. It should be noted that the cell produced biotin here, denoted as Biotin ${ }_{\text {cells }}$, is the same as the biotin term from Equation (6). The change in nomenclature was made to specify the source of biotin within Equation (7).

For the simulation presented here, the following parameters were fit to previously published experimental data [9]: $\mathrm{D}=1.7 \times 10^{-4}, \mathrm{~K}_{4}=1.78 \times 10^{0} \mathrm{~mL} \times \mathrm{ng}^{-1}, \mathrm{~K}_{5}$ $=1.84 \times 10^{0} \mathrm{~mL} \times \mathrm{ng}^{-1}$.

Module three: encapsulated cell-free reactions

Cell-free reactions are frequently used in research, and a reliable model for the transcription and translation dynamics had been previously published in scientific literature [52]. This model relates the concentration of DNA, RNA, and proteins with the availability of transcriptional and translational resources within a cellfree encapsulation. This modeling approach allows one to model cell-free temporal dynamics under varying concentrations of DNA by simulating a set of five ordinary differential equations (Equations (8)-(12)). 


$$
\begin{aligned}
& \frac{\mathrm{d}\left[\mathrm{GFP} \_\mathrm{mRNA}\right]}{\mathrm{dt}}= \mathrm{V}_{6} \times\left(\frac{[\mathrm{TsR}] \times\left[\mathrm{B} \_\mathrm{DNA}\right]}{\mathrm{K}_{6}+\left[\mathrm{B} \_\mathrm{DNA}\right]}\right) \\
&-\delta_{\mathrm{GFP} \_\mathrm{mRNA}} \times\left[\mathrm{GFP} \_\mathrm{mRNA}\right] . \\
& \frac{\mathrm{d}[\mathrm{GFP}]}{\mathrm{dt}}= V_{7} \times\left(\frac{[\mathrm{TIR}] \times\left[\mathrm{GFP} \_\mathrm{mRNA}\right]}{\mathrm{K}_{7}+\left[\mathrm{GFP} \_\mathrm{mRNA}\right]}\right) \\
&-\mathrm{K}_{8} \times[\mathrm{GFP}] . \\
& \frac{\mathrm{d}\left[\mathrm{GFP}{ }^{*}\right]}{\mathrm{dt}}=\mathrm{K}_{8} \times[\mathrm{GFP}] . \\
& \frac{\mathrm{d}[\mathrm{TsR}]}{\mathrm{dt}}=-\delta_{\mathrm{TsR}} \times\left(\frac{[\mathrm{TsR}] \times\left[\mathrm{B} \_\mathrm{DNA}\right]}{\mathrm{K}_{6}+\left[\mathrm{B} \_\mathrm{DNA}\right]}\right) . \\
& \frac{\mathrm{d}[\mathrm{TIR}]}{\mathrm{dt}}=-\delta_{\mathrm{TIR}} \times\left(\frac{[\mathrm{TIR}]}{\mathrm{K}_{9}+[\mathrm{TIR}]}\right) .
\end{aligned}
$$

With Equations (8)-(12), [TsR] and [TIR] represent the concentration of available transcriptional and translational resources respectively. In addition, [GFP*] is the concentration of the fully formed GFP complex that may be monitored optically. Note that the concentrations of GFP encoding mRNA and biotinylated DNA, denoted as [GFP_mRNA] and [B_DNA] respectively, in Equations (8)-(12) are in the cell-free reaction system and independent of the DNA and mRNA expressions from Equations (6) and (7). Furthermore, in the linked system, the concentration of biotinylated DNA is a function of the fraction of streptavidin sites occupied by biotinylated DNA described by Equation (7). The parameters $V_{6}$ and $\mathrm{V}_{7}$ describe the maximum rate of transcription and translation, respectively, whereas $\delta_{\mathrm{GFP} \text { mRNA }}, \delta_{\mathrm{TsR}}$, and $\delta_{\mathrm{TIR}}$ are decay constants for GFP encoding mRNA, transcriptional resources and translational resources, respectively. The parameter values for Equations (8)(12) were previously calculated and used in a model by Stogbauer et al. [52].

Taken together, the models for modules one through three allow us to simulate the cell-free response to inducer chemicals given to engineered cells. All simulations were coded in Python and numerically integrated using RungeKutta based methods within the NumPy library. Data visualization was prepared using the matplotlib library and figures were assembled using Inkscape open-source software. All simulations were performed on a 2012 MacBook Pro running macOS Sierra with a $2.9 \mathrm{GHz}$ Intel Core i7 processor.

\section{ACKNOWLEDGMENTS}

The authors gratefully acknowledge support from award FA9550-13-1-0108 from the Air Force Office of Scientific Research of the USA and award N00014-15-1-2502 from the Office of Naval Research of the USA. The authors additionally acknowledge support from the Institute for Critical Technology and Applied Science at Virginia Polytechnic Institute and State University, from the National Science Foundation Graduate Research Fellowship Program, award number 1607310, and from the Virginia Sea Grant Graduate Research Fellowship Program.

\section{COMPLIANCE WITH ETHICS GUIDELINES}

Keith C. Heyde, MaryJoe K. Rice, Sung-Ho Paek, Felicia Y. Scott, Ruihua Zhang and Warren C. Ruder declare that they have no conflict of interest.

This article does not contain any studies with human or animal subjects performed by any of the authors.

\section{REFERENCES}

1. Ricardo, A. and Szostak, J. W. (2009) Origin of life on earth. Sci. Am., 301, 54-61

2. Szostak, J. W. (2009) Origins of life: systems chemistry on early Earth. Nature, 459, 171-172

3. Szostak, J. W., Bartel, D. P. and Luisi, P. L. (2001) Synthesizing life. Nature, 409, 387-390

4. Adamala, K. P., Engelhart, A. E. and Szostak, J. W. (2016) Collaboration between primitive cell membranes and soluble catalysts. Nat. Commun., 7, 11041

5. Engelhart, A. E., Adamala, K. P. and Szostak, J. W. (2016) A simple physical mechanism enables homeostasis in primitive cells. Nat. Chem., 8, 448-453

6. Gibson, D. G., Glass, J. I., Lartigue, C., Noskov, V. N., Chuang, R. Y., Algire, M. A., Benders, G. A., Montague, M. G., Ma, L., Moodie, M. M., et al. (2010) Creation of a bacterial cell controlled by a chemically synthesized genome. Science, 329, 52-56

7. Hutchison, C. A. III, Chuang, R. Y., Noskov, V. N., Assad-Garcia, N., Deerinck, T. J., Ellisman, M. H., Gill, J., Kannan, K., Karas, B. J., Ma, L., et al. (2016) Design and synthesis of a minimal bacterial genome. Science, 351, aad6253

8. Glass, J. I., Assad-Garcia, N., Alperovich, N., Yooseph, S., Lewis, M. R., Maruf, M., Hutchison C. A. III , Smith, H. O. and Venter, J. C. (2006) Essential genes of a minimal bacterium. Proc. Natl. Acad. Sci. USA, 103, 425-430

9. Zhang, R., Heyde, K. C., Scott, F. Y., Paek, S.-H.and Ruder, W. C. (2016) Programming surface chemistry with engineered cells. ACS Synth. Biol., 5, 936-941

10. Chen, A. Y., Deng, Z., Billings, A. N., Seker, U. O. S., Lu, M. Y., Citorik, R. J., Zakeri, B. and Lu, T. K. (2014) Synthesis and patterning of tunable multiscale materials with engineered cells. Nat. Mater., 13, 515-523

11. Botyanszki, Z., Tay, P. K. R., Nguyen, P. Q., Nussbaumer, M. G. and Joshi, N. S. (2015) Engineered catalytic biofilms: site-specific enzyme immobilization onto E. coli curli nanofibers. Biotechnol. Bioeng., 112, 2016-2024

12. Chen, A. Y., Zhong, C. and Lu, T. K. (2015) Engineering living functional materials. ACS Synth. Biol., 4, 8-11

13. Ridgley, D. M., Freedman, B. G., Lee, P. W. and Barone, J. R. (2014) Genetically encoded self-assembly of large amyloid fibers. Biomater. Sci., 2, 560-566

14. Gardner, T. S., Cantor, C. R. and Collins, J. J. (2000) Construction of a genetic toggle switch in Escherichia coli. Nature, 403, 339-342 
15. Elowitz, M. B. and Leibler, S. (2000) A synthetic oscillatory network of transcriptional regulators. Nature, 403, 335-338

16. Friedland, A. E., Lu, T. K., Wang, X., Shi, D., Church, G. and Collins, J. J. (2009) Synthetic gene networks that count. Science, 324, 1199 1202

17. Anderson, J. C., Voigt, C. A. and Arkin, A. P. (2007) Environmental signal integration by a modular AND gate. Mol. Syst. Biol., 3, 133

18. Ellis, T., Wang, X. and Collins, J. J. (2009) Diversity-based, modelguided construction of synthetic gene networks with predicted functions. Nat. Biotechnol., 27, 465-471

19. Levskaya, A., Chevalier, A. A., Tabor, J. J., Simpson, Z. B., Lavery, L. A., Levy, M., Davidson, E. A., Scouras, A., Ellington, A. D., Marcotte, E. M., et al. (2005) Synthetic biology: engineering Escherichia coli to see light. Nature, 438, 441-442

20. Bashor, C. J., Helman, N. C., Yan, S. and Lim, W. A. (2008) Using engineered scaffold interactions to reshape MAP kinase pathway signaling dynamics. Science, 319, 1539-1543

21. Kramer, B. P., Viretta, A. U., Baba, M. D. -E., Aubel, D., Weber, W. and Fussenegger, M. (2004) An engineered epigenetic transgene switch in mammalian cells. Nat. Biotechnol., 22, 867-870

22. Blake, W. J., Balázsi, G., Kohanski, M. A., Isaacs, F. J., Murphy, K. F., Kuang, Y., Cantor, C. R., Walt, D. R. and Collins, J. J. (2006) Phenotypic consequences of promoter-mediated transcriptional noise. Mol. Cell, 24, 853-865

23. Eldar, A. and Elowitz, M. B. (2010) Functional roles for noise in genetic circuits. Nature, 467, 167-173

24. Guet, C. C., Elowitz, M. B., Hsing, W. and Leibler, S. (2002) Combinatorial synthesis of genetic networks. Science, 296, 1466-1470

25. Kærn, M., Elston, T. C., Blake, W. J. and Collins, J. J. (2005) Stochasticity in gene expression: from theories to phenotypes. Nat. Rev. Genet., 6, 451-464

26. Murphy, K. F., Adams, R. M., Wang, X., Balázsi, G. and Collins, J. J. (2010) Tuning and controlling gene expression noise in synthetic gene networks. Nucleic Acids Res., 38, 2712-2726

27. Balázsi, G., van Oudenaarden, A. and Collins, J. J. (2011) Cellular decision making and biological noise: from microbes to mammals. Cell, 144, 910-925

28. Elowitz, M. B., Levine, A. J., Siggia, E. D. and Swain, P. S. (2002) Stochastic gene expression in a single cell. Science, 297, 1183-1186

29. Karzbrun, E., Tayar, A. M., Noireaux, V. and Bar-Ziv, R. H. (2014) Programmable on-chip DNA compartments as artificial cells. Science, $345,829-832$

30. Noireaux, V., Maeda, Y. T. and Libchaber, A. (2011) Development of an artificial cell, from self-organization to computation and self-reproduction. Proc. Natl. Acad. Sci. USA, 108, 3473-3480

31. Shimizu, Y., Inoue, A., Tomari, Y., Suzuki, T., Yokogawa, T., Nishikawa, K. and Ueda, T. (2001) Cell-free translation reconstituted with purified components. Nat. Biotechnol., 19, 751-755

32. Tan, C., Saurabh, S., Bruchez, M. P., Schwartz, R. and Leduc, P. (2013) Molecular crowding shapes gene expression in synthetic cellular nanosystems. Nat. Nanotechnol., 8, 602-608

33. Weber, P. C., Ohlendorf, D. H., Wendoloski, J. J. and Salemme, F. R. (1989) Structural origins of high-affinity biotin binding to streptavidin. Science, $243,85-88$

34. Green, N. M. (1963) Avidin. 3. The nature of the biotin-binding site. Biochem. J., 89, 599-609

35. Huang, S. -C., Stump, M. D., Weiss, R. and Caldwell, K. D. (1996) Binding of biotinylated DNA to streptavidin-coated polystyrene latex: effects of chain length and particle size. Anal. Biochem., 237, 115-122

36. Noireaux, V., Bar-Ziv, R. and Libchaber, A. (2003) Principles of cellfree genetic circuit assembly. Proc. Natl. Acad. Sci. USA, 100, 12672 12677

37. Daube, S. S. and Bar-Ziv, R. H. (2013) Protein nanomachines assembly modes: cell-free expression and biochip perspectives. Wiley Interdiscip. Rev. Nanomed. Nanobiotechnol., 5, 613-628

38. Groisman, A., Lobo, C., Cho, H., Campbell, J. K., Dufour, Y. S., Stevens, A. M. and Levchenko, A. (2005) A microfluidic chemostat for experiments with bacterial and yeast cells. Nat. Methods, 2, 685-689

39. Hol, F. J. H. and Dekker, C. (2014) Zooming in to see the bigger picture: microfluidic and nanofabrication tools to study bacteria. Science, 346, 1251821

40. Sun, Z. Z., Hayes, C. A., Shin, J., Caschera, F., Murray, R. M. and Noireaux, V. (2013) Protocols for implementing an Escherichia coli based TX-TL cell-free expression system for synthetic biology. J. Vis. Exp., doi: 10.3791/50762

41. Lutz, R. and Bujard, H. (1997) Independent and tight regulation of transcriptional units in Escherichia coli via the LacR/O, the TetR/O and AraC/I1-I2 regulatory elements. Nucleic Acids Res., 25, 1203-1210

42. Sanyal, I., Cohen, G. and Flint, D. H. (1994) Biotin synthase: purification, characterization as a $[2 \mathrm{Fe}-2 \mathrm{~S}]$ cluster protein, and in vitro activity of the Escherichia coli bioB gene product. Biochemistry, 33, 3625-3631

43. Brophy, J. A. N. and Voigt, C. A. (2014) Principles of genetic circuit design. Nat. Methods, 11, 508-520

44. Garcia-Ojalvo, J., Elowitz, M. B. and Strogatz, S. H. (2004) Modeling a synthetic multicellular clock: repressilators coupled by quorum sensing. Proc. Natl. Acad. Sci. USA, 101, 10955-10960

45. Heyde, K. C. and Ruder, W. C. (2015) Exploring host-microbiome interactions using an in silico model of biomimetic robots and engineered living cells. Sci. Rep., 5, 11988

46. Anderson, J. C., Voigt, C. A. and Arkin, A. P. (2007) Environmental signal integration by a modular AND gate. Mol. Syst. Biol., 3, 133

47. Cameron, D. E. and Collins, J. J. (2014) Tunable protein degradation in bacteria. Nat. Biotechnol., 32, 1276-1281

48. Gardner, T. S., Cantor, C. R. and Collins, J. J. (2000) Construction of a genetic toggle switch in Escherichia coli. Nature, 403, 339-342

49. Johnson, K. A. and Goody, R. S. (2011) The original Michaelis constant: translation of the 1913 Michaelis-Menten paper. Biochemistry, 50, 8264-8269

50. González, M., Bagatolli, L. A., Echabe, I., Arrondo, J. L. R., Argaraña, C. E., Cantor, C. R. and Fidelio, G. D. (1997) Interaction of biotin with streptavidin. Thermostability and conformational changes upon binding. J. Biol. Chem., 272, 11288-11294

51. Schwarz-Schilling, M., Aufinger, L., Mückl, A. and Simmel, F. C. (2016) Chemical communication between bacteria and cell-free gene expression systems within linear chains of emulsion droplets. Integr. Biol., 8, 564-570

52. Stögbauer, T., Windhager, L., Zimmer, R. and Rädler, J. O. (2012) Experiment and mathematical modeling of gene expression dynamics in a cell-free system. Integr. Biol., 4, 494-501

53. Brenner, K., You, L. and Arnold, F. H. (2008) Engineering microbial consortia: a new frontier in synthetic biology. Trends Biotechnol., 26, 483-489

54. Hays, S. G., Patrick, W. G., Ziesack, M., Oxman, N.and Silver, P. A. (2015) Better together: engineering and application of microbial symbioses. Curr. Opin. Biotechnol., 36, 40-49 
55. Wintermute, E. H. and Silver, P. A. (2010) Dynamics in the mixed microbial concourse. Genes Dev., 24, 2603-2614

56. Balagaddé, F. K., Song, H., Ozaki, J., Collins, C. H., Barnet, M., Arnold, F. H., Quake, S. R.and You, L. (2008) A synthetic Escherichia coli predator-prey ecosystem. Mol. Syst. Biol., 4, 187

57. Heyde, K. C., Gallagher, P. W. and Ruder, W. C. (2016) Bioinspired decision architectures containing host and microbiome processing units. Bioinspir. Biomim., 11, 056017

58. Tran, H., Oliveira, S. M. D., Goncalves, N. and Ribeiro, A. S. (2015)
Kinetics of the cellular intake of a gene expression inducer at high concentrations. Mol. Biosyst., 11, 2579-2587

59. Xu, H., Moraitis, M., Reedstrom, R. J. and Matthews, K. S. (1998) Kinetic and thermodynamic studies of purine repressor binding to corepressor and operator DNA. J. Biol. Chem., 273, 8958-8964

60. Politi, N., Pasotti, L., Zucca, S., Casanova, M., Micoli, G., Cusella De Angelis, M. G. and Magni, P. (2014) Half-life measurements of chemical inducers for recombinant gene expression. J. Biol. Eng., 8,5 